\title{
MENORES, JÓVENES, EDUCACIÓN, DROGAS Y JUSTICIA
}

\author{
JUUVENILES, YOUNG PEOPLE, EDUCATION, DRUGS AND JUSTICE
}

\author{
Concepción Nieto Morales \\ Universidad Pablo de Olavide, Sevilla. España/Spain \\ concepcionm@upo.es
}

Recibido/Received: 29/06/2012

Modificado/Modified: 12/07/2012

Aceptado/Accepted: 27/09/2012

\section{RESUMEN}

El consumo de drogas se encuentra normalizado en los jóvenes de hoy día, comienzan por el consumo de hachís a temprana edad, algunos se mantienen en el consumo de pequeñas cantidades o abandonan el consumo y otros van incrementando el consumo y ampliando el tipo de sustancias que consumen. Los menores que van acumulando fracaso escolar, no consiguen el nivel del resto de los alumnos ni superación las pruebas académicas, desencadenando expulsiones, ociosidad, relación con grupo de iguales en similares circunstancias pasando la mayor parte del tiempo ociosos se reúnen con grupos de similares circunstancias de ociosidad, conductas disruptivas, y el aburrimiento les lleva a comenzar a consumir hachís, y otro tipo de sustancias estupefacientes que desencadenan adicciones en ocasiones muy graves y problemas judiciales.

\section{PALABRAS CLAVE}

Amigos, consumo estupefacientes, adicciones, delito.

\section{SUMARIO}

1. Introducción. 2. Jóvenes y familia. 3. Los jóvenes y el grupo de iguales. 4. Jóvenes, drogas y educación. 5. Del delito contra la salud pública al consumidor de drogas. 6. Lectura de resultados. 7. A modo de conclusión. Bibliografía

\begin{abstract}
Drug consumption has been normalized for young people nowadays. They start consuming hashish in an early age. Some of them keep that consumption with small amounts or quit, and others increase it and even enlarge the range of substances to consume. Those juveniles accumulating school failure can't reach the rest of students' level and can't pass the academic tests, which results in expulsions, leisure time and relationships with other individuals in the same circumstances: they spend most of the time idle, gathering in groups in the same circumstances of idleness and disruptive conducts. Boredom leads them to start consuming hashish and other narcotic substances, which ends sometimes in very serious addictions, and judicial problems.
\end{abstract}

\section{KEYWORDS}

Friends, consumption, narcotics, addictions, offence.

\section{CONTENTS}

1. Introduction. 2. Juveniles and family. 3. Juveniles and the group of equals. 4. Juveniles, drugs and education. 5. From the crime against public health to the consumption of drugs. 6 . Reading results. 7. In conclusion. References 


\section{INTRODUCCIÓN}

No cabe ninguna duda que los menores y jóvenes son el futuro y hay que cuidarles, educarles, en definitiva proporcionarles un camino de futuro en las mejores condiciones posibles. Los diferentes conceptos que trataremos en este artículo serán objeto de un análisis para centrar el tema de estudio.

La edad es el tiempo que se ha vivido, está recogida en el ordenamiento jurídico y combina la capacidad de obrar general con otras capacidades, marcando la frontera entre la minoría y mayoría de edad.

El concepto de menor es relativo, la infancia, se refleja en múltiples instrumentos y convenios, con especial referencia a los Derechos que se le atribuyen.

La edad y los derechos de los menores y jóvenes, se encuentran recogidos y se protegen sus derechos en diferentes ámbitos legales, como Constitución Española (1978) la Convención de las Naciones Unidas (1989), el Código Penal (1995), Código Civil (1889), y otras leyes como la LORPM (5/2000; 8/2006), etc.

La Convención de los Derechos del Niño reúne los Derechos Humanos de la Infancia, proporcionando derechos desde principios rectores al concepto que tenemos de infancia. La Convención de las Naciones Unidas en su artículo primero define al niño como "todo ser humano menor de dieciocho años, salvo que, en virtud de la ley que le sea aplicable, haya alcanzado antes la mayoría de edad" (CNU, 1989).

En el Informe de la Juventud en España se entiende por joven a las personas cuya edad se encuentra comprendida entre los 16 y los 29 años (López et al, 2005).

Los mayores de 14 años que sean imputados por sus actos que sean calificados en el Código Penal como delitos o faltas, se les juzgará a tenor de la Ley Orgánica de Responsabilidad Penal del Menor (LORPM) que fija la edad de actuación para aplicarse a los mayores de 14 y menores de 18 años. El Código Penal (1995) y la LORPM, se complementan siendo la LORPM sancionadora educativa, considerando que el menor no ha tenido tiempo de adquirir psíquica y culturalmente las pautas de comportamiento social necesarias para ser considerado plenamente responsable de sus actos; dicha ley cuando el menor ha cumplido los 18 años se refiere a él como joven.

La definición más utilizada de droga es "toda sustancia que, introducida en el organismo por cualquier vía de administración, produce una alteración de algún modo, del natural funcionamiento del sistema nervioso central del individuo y es, además, susceptible de crear dependencia, ya sea psicológica, física o ambas." (Kramer, у Cameron, 1975:13). Son sustancias naturales o sintéticas, legales o ilegales cuyo consumo excesivo o prolongado produce dependencia además de afectación física, psicológica, social, etc.

El consumo de sustancias estupefacientes y concretamente el de cannabis ha aumentado de forma considerable ente los adolescentes españoles según el Informe del Plan Nacional sobre Drogas, siendo la droga ilegal que más se consume en España y en el mundo (Informe Plan Nacional de drogas, 2007), es la más consumida por la juventud en España Para los jóvenes fumarse un porro es algo tan normal como si fuese tabaco. Desde los Servicios sociales comunitarios, los más cercanos a los usuarios, existen programas de prevención del consumo de estupefacientes, y es fundamental la escucha al usuario, como manifiesta González Resa (2009), y demuestra proximidad a la vez que establecer relaciones y vínculos que permitan intervenir a los profesionales. 
Se ha realizado un estudio cualitativo en Centros de internamiento mediante la técnica del grupo de discusión para conocer el discurso de los menores que han tenido contacto y consumo con sustancias estupefacientes y que en el momento de su ingreso en el Centro terapéutico, se encontraban con gran descontrol por la excesiva ingesta de estupefacientes sin ser conscientes de su situación.

\section{JÓVENES Y FAMILIA}

Cuando nacemos, el destino se encarga de situarnos en una familia de origen que será la encargada de educarnos, proporcionar al niño cariño y apego, acompañarnos hasta que seamos personas adultas y autónomas. El concepto de familia, su organización y estructura, prácticas educativas, etc., que nos transmita la socialización, tendrá gran influencia en nuestro comportamiento y marcará nuestra vida presente y futura (Taberner Güas, 2012)

Los menores que han vivido en medio de pautas de consumo han adquirido una interiorización de normalidad sobre conductas con las drogas que influirá directamente sobre su futuro.

El consumo de sustancias estupefacientes es uno de los graves problemas que existen en el mundo y mueve mucho dinero directa e indirectamente, legal e ilegal lo que hace que sea tan difícil de erradicar. Las estrategias de prevención pasan por implicar a instituciones desde la familia, escuela, administración, etc.

Si hasta hace relativamente poco tiempo se consideraba que el consumo de drogas era un problema mayoritariamente de grupos deprimidos y marginales, esa tendencia ha cambiado, al tiempo que lo ha hecho la sociedad.

La familia como unidad básica de socialización es el pilar fundamental para que los menores adquieran valores que les permitan un futuro normalizado, pero la familia sola no puede educar y es la escuela que además contribuye a esta socialización secundaria facilitando la integración, siendo muy importante también el aprendizaje social. Todos estos ingredientes son los que permiten que los menores y jóvenes puedan tener una vida personal y social normalizada. Para los menores y jóvenes la educación tanto formal como informal tiene gran impacto en su futuro permitiendo su integración social y laboral o evitando que entre o se perpetúen en la espiral de la exclusión.

La influencia del aprendizaje social incide en gran medida para adquirir pautas de conducta de consumo de droga. El clima familiar en el hogar con adolescentes es un elemento clave para predecir el consumo de sustancias, siendo los padres los que deben situar el marco normativo de los hijos y a medida que estos crecen y se desarrollan, este marco normativo va siendo modificado (Elzo, 2003). A medida que aumenta el consumo de sustancias ilegales en los jóvenes las relaciones familiares se deterioran debido, en gran medida, a que el consumo altera el normal desarrollo de sus hábitos debido a querer ocultar la ingesta de estupefacientes.

El funcionamiento familiar y el clima de relación entre los padres y los diferentes miembros de la familia es uno de los factores que más influencia tiene en los hijos. Con los cambios sociales, la estructura familiar ha sufrido importantes modificaciones y en muchos casos pérdida de valores fundamentales para la educación de los hijos.

La violencia y delincuencia no son hechos sociales aislados, se encuentran inmersos en contextos sociales mucho más complejos, imbricados en estructuras sociales, económicas, 
legales, políticas, etc. La violencia social y la delincuencia tienen raíces en contextos ideológicos y de sistema de valores que cuestiona al modelo social actual.

El contexto social predispone e incluso puede fomentar la exclusión: el sistema genera periferias sociales, económicas, culturales y hasta cierto punto institucionalización, aceptando su existencia y se hace cargo de ellas (Tourain, 2006) El sistema económico domina y sitúa al margen y excluye creando situaciones y economías marginales ante necesidades y realidades toleradas.

Victoria Camps (2008) reafirma la idea de que la formación corresponde, sobre todo, a la familia complementada con la escuela sin olvidar el aprendizaje social a los que contribuyen los medios de comunicación, las redes sociales y el espacio público en todas sus manifestaciones. Considera urgente recuperar valores como el respeto, la convivencia, el esfuerzo, la equidad o la utilización razonable de la libertad y conceptos como la autoridad, normas, esfuerzo, disciplina y tolerancia, etc., debiendo cambiar de perspectiva, eliminar tópicos y asumir que hay que enseñar y revalorizar estos valores.

"No podemos inhibirnos de la responsabilidad colectiva que supone educar. El futuro y el bienestar de la sociedad dependen de nuestro compromiso" (Camps, 2008:45).

El consumo y "coqueteo" de drogas en el momento actual no son percibidas por los jóvenes como un problema, sin ser conscientes de que el consumo diario de cannabis puede predisponer a la aparición de psicosis y esquizofrenia. Aquellos episodios de psicosis que son fruto de estas sustancias estupefacientes, presentan unas características específicas tanto antes de aparecer como en la presentación clínica y repercusiones futuras en consumidores (Ruiz Veguilla, 2008).

"El uso regular entre los menores de 15 años se encuentra más a menudo en un grupo de la población altamente problemático, en el que el uso de sustancias está asociado con otros trastornos psicológicos y sociales. El cannabis actúa sobre las funciones de la memoria, la atención y la percepción, así como la actividad motora. De este modo al consumir cannabis todas estas funciones se ven alteradas es más difícil mantener la atención y recordar eventos y la percepción de la realidad se ve alterada, siendo también difícil la estimación del paso del tiempo (Otero, 2009:157).

\section{LOS JÓVENES Y EL GRUPO DE IGUALES}

La adolescencia es una etapa donde el joven afianza su relación con el grupo de iguales y en la que se va desligando de esa identidad familiar afirmando su personalidad. En la elección del grupo de iguales influyen gran cantidad de factores que presentan muchos condicionantes; los jóvenes residentes en barrios deprimidos y marginales, donde el consumo de drogas es una cuestión normalizada, tienen mayores posibilidades de comenzar a consumir que los que residen en zonas normalizadas.

"En la etapa adolescente, el grupo de amigos es un marco imprescindible para consolidar la identidad individual y social de los jóvenes. El grupo les va a permitir identificarse con aquellos y aquellas que están pasando por su misma situación, pudiendo reconocerse a sí mismos en este entorno y recibiendo apoyo desde esta comprensión mutua. A cambio de este apoyo, el grupo les exige el seguimiento de unas normas, 
ejerciendo influencia en el individuo. En la medida en que la familia y la escuela no tengan un peso específico, el grupo aumentará su influencia y hará que éste dependa excesivamente de él. De esta forma, el consumo o no de drogas por parte de los amigos puede influir en el consumo propio” (Otero, 2009:159).

La escolaridad es obligatoria hasta los 16 años, cuando la familia no valora adecuadamente la formación, presentan absentismo, bajo rendimiento, abandono y entran en espirales de acciones que les están condicionando y en muchas ocasiones incitan para comenzar a consumir. Diferente es la situación de jóvenes que pertenecen a familias de clase media y media alta donde la relación de los jóvenes con su grupo de iguales, de familias en situaciones similares, aunque por otras circunstancias, son las que les introduce en el consumo de sustancias adictivas.

El bajo rendimiento y aburrirse en la escuela puede provocar conflictos que lleven a expulsiones y que en horario escolar esos jóvenes se encuentren bien delante del ordenador interrelacionando con otros jóvenes en similares circunstancias que se encuentran en un periodo de expulsión escolar o bien ya han abandonado la escolaridad aunque su edad es de escolaridad obligatoria; y en otras ocasiones presentan absentismo escolar bien con conocimiento de los padres o sin él, lo que les permiten estar más tiempo en la calle ociosos, cuando deberían de estar en la escuela por ser horario escolar y relacionándose con otros jóvenes en similares circunstancias ideando actividades en muchos casos por aburrimiento y en otras ya de forma consciente y bien se inician el consumo de hachís o bien afianzan dicho consumo. Son situaciones problemáticas y a veces se rozan situaciones marginales. Estas y otras situaciones igual de graves se viven a diario cuando por sus acciones los jóvenes entran en conflicto con la ley y deben acudir al ámbito judicial y en las exploraciones que se les realizan en los equipos psico-socioeducativos de la Fiscalía de la jurisdicción de Menores se detectan estas problemáticas.

"La educación de los últimos años no ha sabido transmitir el valor del esfuerzo. Las razones son variadas y seguramente más complejas de lo que seré capaz de detallar en estas páginas. Hay una cuestión que apunta la causa de muchos de los despropósitos educativos que empezamos a lamentar. Me refiero a la importancia dada a la motivación para el estudio y para todo aquello que cuesta llevar a la práctica. En la escuela es donde el alumno se habitúa de forma sistemática a esforzarse y a trabajar; la voluntad de motivar ha sustituido a la voluntad de inculcar actitudes o modos de hacer las cosas. No es ningún descubrimiento constatar que a los niños no les gusta estudiar, les cuesta mantener la atención, escuchar al maestro y aguantar toda una clase sin moverse ni enredar..." (Camps, 2008:95).

En una sociedad fragmentada en minorías, sin contacto o con deficiente contacto cara a cara, donde las relaciones sociales se desarrollan en otros ámbitos, como las redes de interacción social virtuales (Twitter, Facebook, etc.), donde el intercambio entre personas y grupos en diferentes contextos, a veces muy complejos, no requieren conocerse y al principio muchos no se conocen al residir en un radio más o menos alejado pero que permite el desplazamiento, involucra a jóvenes en relaciones que, a veces, aunque no tengan intereses comunes en aprendizaje formal, ni estatus social, sí que comparten aprendizaje informal y se relacionan en actividades de ocio que permite a minorías aisladas y discriminadas relacionarse con otras personas que en algunos de los casos les inician en el consumo de sustancias estupefacientes. 
Las redes sociales no se pueden considerar buenas o malas en sí mismas, son una forma de relación en auge debido a la nueva estructura social, ofrecen un mundo diferente en sí mismo, son conexión y comunicación entre usuarios, debiéndose asumir la responsabilidad personal porque a veces ofrecen expectativas que no se pueden ver cumplidas debiendo cada persona velar por su propia seguridad. Esta cuestión es responsabilidad en los menores de edad de las figuras adultas de referencia para velar como responsables de su seguridad. Es importante no generalizar en estos aspectos y controlar al menor de edad, lo que en ocasiones resulta imposible para muchos padres y tutores debido a la brecha digital existente entre la generación de padres y responsables y los hijos. La personalidad de las figuras adultas de referencia para los jóvenes y las vivencias que estos han interiorizado en la familia y escuela se encuentra íntimamente interrelacionado con las acciones que estos realizan (Camps, 2008; Nieto-Morales, González Lara, 2012).

\section{JÓVENES, DROGAS Y EDUCACIÓN}

Existen numerosos estudios que relacionan el fracaso escolar con el consumo de estupefacientes (Luengo, et al., 1996; Nieto-Morales, 2005; Gallardo Fernández, NietoMorales, 2010). La escuela, como segunda institución fundamental de socialización de los menores, presenta una incidencia fundamental, y de la buena integración de los jóvenes en la institución educativa va a depender su mayor o menor rendimiento académico. Factores tanto intrínsecos como extrínsecos influirán en el rendimiento académico de los alumnos, a veces el clima familiar presenta tanta influencia que imposibilita a la escuela poder contribuir de forma adecuada en la formación de estos alumnos. La disonancia educativa entre padres y profesores e institución escolar dificulta la educación de los menores y jóvenes. Aunque la escuela intente realizar una labor compensatoria de las desigualdades, no siempre se consigue el objetivo si no existe colaboración por parte de las demás instituciones implicadas en la labor. Las buenas relaciones entre padres e institución educativa son de una gran importancia para el buen rendimiento académico de los alumnos; cuando por alguna circunstancia faltan, fallecimiento, separación, ausencia, etc., tiene relación directa en el descenso de los resultados académicos que obtienen. (Elzo, 2003:216-228).

El consumo de drogas de los estudiantes de secundaria, entre los 14 y 18 años, se vincula al ocio y al fin de semana. Entre ellos se encuentran alumnos con bajo rendimiento que presentan expulsiones y que finalmente llegan a abandonar la enseñanza obligatoria antes de obtener la certificación académica cuando cumplen los 16 años o incluso mucho antes de esta edad incurren en el consumo y conductas violentas que en muchos casos les lleva a presentar conflicto con la ley vigente. (Martínez-Lorca, Alonso-Sanz, 2003:146)

La práctica profesional de los profesores de educación secundaria, que es el colectivo profesional más directamente relacionado con los jóvenes estudiantes, tiene como observatorio el comienzo del consumo de estupefacientes en muchos jóvenes escolares. Muchos profesores ven la institución donde desempeñan su trabajo como conflictiva y un número considerable se encuentra con problemas de salud debido a los conflictos que perciben en el desempeño de su trabajo.

Elevar la edad de enseñanza obligatoria y las diferentes leyes que la consolidaron han contribuido a que la escuela se considere conflictiva, además de que la escala de valores ha cambiado y se han relajado los valores necesarios que deben imperar, deteriorando en gran medida las pautas educativas. La autoridad está desvalorizada sino eliminada, unida al 
cambio en la estructura familiar que deteriora su funcionamiento. La escala de valores imperante es relativa y la catedrática de filosofía Victoria Camps así lo manifiesta:

“...Las causas que la juventud defiende, como el ecologismo y el respeto por la naturaleza no son en realidad una prioridad vital (....) en estos jóvenes hay una falla entre los valores finalistas y los valores instrumentales: los jóvenes de hoy apuestan por invertir en pacifismo, tolerancia, ecología, exigencia de lealtad, etc., y a la vez. presentan fallas en valores instrumentales sin los cuales los valores anteriores corren el riesgo de quedarse en un bonito discurso. Son déficit que presentan los valores como el esfuerzo, la responsabilidad, la participación, la abnegación, la aceptación del limite, el trabajo bien hecho.... (...)." (Camps, 2008:102)

Los jóvenes no tienen conciencia de las responsabilidades que les confiere su estatus, se convierten en adultos a una edad sin responsabilidad y con deficiencias en sus valores personales, sociales y educativos, considerando que el valor de la meritocracia y esfuerzos personales no son importantes, situación que se traduce en aprovechar el instante vital presente sin tener que realizar ningún tipo de esfuerzo. Estas situaciones son transmitidas en las entrevistas realizadas para valorar las circunstancias socioeducativas enmarcadas en la LORPM $(5 / 2000 ; 8 / 2006)$

La experiencia en el desempeño laboral, nos afirma que el consumo de estupefaciente se convierte en una forma de tragedia, debido a que con él baja aun más el rendimiento académico y en su caso laboral, tanto que los padres, educadores y profesionales de la salud ante tal situación se encuentran preocupados por la espiral de consumo y en algunas ocasiones van encontrando la situación normal porque van asumiendo este consumo como normal. El consumo de drogas desencadena, más tarde o más temprano, violencia y si continúan en el ámbito escolar esta también se produce en la institución educativa con la consiguiente alteración y agresiones tanto a compañeros como profesores.

\section{DEL DELITO CONTRA LA SALUD PÚBLICA AL CONSUMIDOR DE DROGAS}

¿Qué se entiende por consumo?, ¿Cuándo el consumo es ilegal?

Consumo es aquel uso que implique la aplicación de la sustancia a la persona. La relación entre drogodependencias y problemas jurídicos o delito es un binomio que se encuentra presente en la mayor parte de los temas relacionados con las drogas, ya sea consumo o tráfico. Los mayores problemas se encuentran en los consumidores ya que llegan a tener problemas con la justicia. El consumo conlleva un coste económico para mantenerlo que obliga al consumidor a terminar incurso en distintas formas de delincuencia, salvo que exista gran solvencia económica. Se distinguen dos tipos de delitos: los que se llevan a cabo para conseguirla y los que se producen como consecuencia del consumo (Álvarez et, al, 2009).

El tráfico de drogas requiere un mercado ilegal con peculiaridades y sus propias normas y un valor añadido importante, todo ello muy diferente al mercado legal de productos. La venta de estupefacientes proporciona un dinero fácil y, a pequeña escala, suelen hacerlo personas necesitadas de ingresos que tienen dificultades para conseguir otro tipo de trabajo y aprovechan poder conseguir un dinero fácil sin tener en cuenta las consecuencias que pueden acarrear, incluidos los gastos judiciales, policiales, reinserción, agravio para el erario público, etc. 
El vínculo entre consumo de drogas y delincuencia es que el 51\% de hombres y $69 \%$ de mujeres reclusos, refieren que al cometer el delito estaban bajo influencia de las drogas. El tráfico de drogas y blanqueo de capitales se encuentra íntimamente relacionado. Existe cooperación internacional. La inviolabilidad del domicilio es un derecho fundamental recogido en el artículo 18.2 de la Constitución y ninguna entrada o registro podrá hacerse sin el consentimiento del titular o resolución judicial salvo caso de flagrante delito.

El consumo de drogas en España no está tipificado como delito, sin embargo el tráfico es considerado un delito contra la salud pública, el lavado de dinero, considerándose este como procedente de venta, ganancias económicas de la venta de estupefacientes (Romero Casanova, 2006).

La falta de conocimiento sobre los efectos del consumo de drogas sobre la conducción de vehículos al igual que el desconocimiento de los efectos de cierto tipo de medicamentos que puede provocar disminución de la capacidad para conducir, lleva a que sean múltiples los accidentes bajo los efectos del alcohol, drogas tóxicas, estupefacientes y sustancias psicotrópicas, así como de esos medicamentos.

Las conductas del consumidor de estupefacientes se ven deteriorada por el consumo. Dado que el consumo de estupefacientes genera dependencia física y síndrome de abstinencia y conlleva tener que obtener dinero para el consumo, lo que produce que los consumidores presenten un elevado riesgo de realizar conductas antisociales, que suelen ser de dos tipos, las relacionadas con la obtención de la propia sustancia para consumirla y recurrir a la compraventa por lo que se consideraría traficante estando tipificado como delito Código Penal (1995).

En el tratamiento jurídico penal se trata de articular mecanismos nacionales e internacionales para combatir el tráfico de drogas. En los resortes de combate a gran escala se encuentran, el Convenio Naciones Unidas sobre Estupefacientes (2008), y el Convenio de Sustancias Psicotrópicas. También a nivel de la Unión Europea se lucha contra la delincuencia organizada: Ley 1993; Blanqueo de capitales; Ley, 2003, Investigación ámbito europeo sobre drogas, etc. En el ámbito español, el Código Penal (1995) tipifica la represión penal del tráfico de drogas, aunque existen antecedentes y reformas legislativas posteriores, respecto a los delitos contra la salud pública, drogas tóxicas o estupefacientes, etc.

Las sanciones del llamado tráfico ilegal de drogas se refleja en numerosos artículos del Código Penal español vigente (1995), penando el cultivo y elaboración; existe variación de la pena según la sustancia estupefaciente; aunque se recogen conductas atípicas (autoconsumo, consumo compartido, insignificancia) y otras de extrema gravedad.

El tráfico de drogas generalmente a gran escala lleva aparejado el blanqueo de dinero y ganancias obtenidas con su conversión o blanqueo, considerándose delito. En todo Proceso Judicial el reflejo del delito conlleva la presunción de inocencia hasta que la acusación en el acto de Audiencia aporte las pruebas de cargo, constitucionalmente obtenidas, legalmente practicadas y valoradas, correspondiendo al Tribunal (Art., 741 Ley Criminal) la resolución Judicial de dictar Sentencia.

Considerándose que el tráfico de drogas a pequeña escala o menudeo es imprescindible combatirlo, dado que por él pasa toda la droga y el dinero, y se expande la toxicomanía, creando nuevos adictos, y su impunidad produce más consumidores, vendedores, etc. Para combatir dicho tráfico, la policía encuentra ambientes hostiles, falta de colaboración, estructura de la ubicación dificultosa, falta de información, etc. El trabajo policial a nivel de menudeo es muy complicado, debiendo seleccionar sus objetivos, realizar vigilancias y planificar minuciosamente además de obtener los mandamientos judiciales para entradas y 
registros, etc. El tráfico de drogas a gran escala, según la Junta Internacional de Fiscalización de Estupefacientes, generalmente procede de la zona rural de Marruecos, siendo muy rentable su cultivo en el país de origen, ya que los cultivos alternativos son un fracaso (JIFE, 2010). Las incautaciones de hachís en España han ascendido en los últimos años, a pesar de las nuevas formas de introducir la droga.

Según las informaciones que recoge el Informe Mundial sobre las Drogas (ONU, 2008), 208 Millones de personas en el mundo consumen droga al menos una vez al año. Precisa que para 26 millones de personas, es decir, el 0,6 por ciento de la población adulta del planeta, la droga supone un serio problema. Estas informaciones nos permiten analizar y concienciarnos, existiendo numerosos avances, estudios, noticias, etc., de este problema tan importante que existe a nivel mundial.

\section{LECTURA DE RESULTADOS}

Se realizan grupos de discusión con jóvenes bajo medidas judiciales con sentencias en firme de condena por el Juez de Menores y que se encuentran internos en Centros de internamiento de la comunidad autónoma de Andalucía. Los grupos de discusión se han realizado siguiendo la pautas de investigación científica (Ibáñez, 1986)

Desde la promulgación de la LORPM 5/2000 de 13 de enero, y su modificación por la LORPM 8/2006 de 4 de diciembre se han dedicado recursos para la ejecución de las medidas que prevé la ley y los recursos de los Centros existentes se han ido adaptando a las necesidades y demandas y así han mejorando. Se crea en algunos Centros módulos para tratamientos terapéuticos adaptados a las demandas sobre la ejecución de medidas de internamiento para tratamiento terapéutico de menores consumidores de drogas. Se realizan grupos de discusión de menores que están cumpliendo medida de internamiento en Centros y recibiendo tratamiento terapéutico.

Estos menores y jóvenes, condenados por hechos delictivos graves que además presentan adicciones graves, son chicos con perfiles psicosociales y características especiales en general bastante similares.

Caso 1: Joven de 20 años, cumple una medida de internamiento en Centro terapéutico por un delito de robo. Ha cumplido medidas anteriores y también tiene medidas pendientes de ejecución. Abandonó la escolaridad cuando cursaba el primer curso de la ESO. Pertenece a una familia mono-parental y a veces oscila a la familia extensa siendo colaboradora y visitándole en el Centro. El padre falleció y la madre es empleada del hogar. Son 3 hermanos donde él ocupa el primer lugar en orden cronológico. La familia reside en un barrio urbano problemático. Es consumidor de hachís, cocaína, drogas sintéticas, alcohol heroína. Respecto a la valoración del Centro sobre la probabilidad de futura reinserción la califican como positiva.

El joven, mantiene un discurso aflorando sus amargas vivencias familiares.

"Mis padres tenían problemas con la droga, desde pequeño lo que he visto en mi casa ha sido droga, siempre mucha gente rara, muchas peleas en mi casa, mi madre se escapó conmigo a Inglaterra y mi padre fue a buscarnos cuando salió de la cárcel, he visto jeringuillas, y yo qué sé..., pastillas, de todo. Siempre había problemas en mi casa, mi padre entraba y salía de prisión constantemente, mi madre es inglesa y vino a 
España con 3 años, yo nací cuando ella tenía 17 años, cuando cumplí 8 años mis padres se separaron. Me quedé con mi madre pero yo no quería estar con ella, quería estar con mi abuelo paterno, que era el que me compraba las cosas, me paseaba con el coche, etc., mi madre no podía conmigo desde que tenía 9 años y dejé de ir al colegio, a fumar porrillos a escondidas con otros chavales, con 11 años una rayita, que no sabía ni que era eso. A partir de los 13 comencé el consumo fuerte y a robar para conseguir dinero para consumir. A los 16 años me metieron en el primer centro, mi padre ya estaba rehabilitado y me reñía a lo que yo le respondía que le había visto hacer de todo, "tú a mi qué me vas a decir ...". Estuvo 8 meses en el primer centro (...) que no "me sirvió de nada, porque seguía consumiendo y robando", estuvo trabajando y drogándose. Falleció mi padre y otra vez todo se fue al garete. Este internamiento me ha venido bien porque si no hubiera seguido, estaría peor, las relaciones eran muy malas con la familia, había amenazado a mi madre con un cuchillo, y a los vecinos, "se ciega uno" y no sabe hasta dónde puede llegar, le robaba "empastillao", robando constantemente, mis vecinos no me denunciaban porque les daba pena. Me llevaron a un centro a Málaga y a los 3 meses me trasladaron a otro centro, tenía orden de alejamiento de la madre, ahora estoy muy bien con ella, le veo sentido a la vida y me lo paso bien sin drogas, me relaciono con otros amigos, paso de gente con problemas, los amigos un sábado por la noche en una discoteca se toman una copa pero eso es normal, lo que no es normal es consumir drogas. Le veo sentido a la vida y me he dado cuenta que no hace falta drogarse para pasárselo bien" (Caso 1, 20 años).

Caso 2: 20 años. Cumple una medida de internamiento en un Centro terapéutico por un delito de robo. Tiene pendiente de cumplimiento mas medidas. Abandonó la escolaridad cuando cursaba el primer curso de la ESO. Pertenece a una familia estructurada y colaboradora que le visita en el Centro. El padre es periodista y su madre administrativa, son dos hermanos y el joven es el primogénito. La familia reside en una zona urbana residencial. Es consumidor de hachís, cocaína, pastillas y alcohol. La valoración del Centro resalta buenas perspectivas de reinserción.

El joven es actualmente mayor de edad con estancia en Centro terapéutico para desintoxicación y arroja un discurso pragmático y realista

"El que hace algo es porque quiere, el grupo tiene drogadictos y algunos que no consumen ni roban, los que consumen no ven el problema y si consumen delante de nosotros nos ponen en riesgo, en el fondo no ven el problema que representan. No todo es de color de rosa, algunos basta que te vean bien para que quieran que vuelvas a caer ofreciéndote e incitándote". El barrio tiene zonas que pueden consumir, hoy en cualquier zona consumen.

Mi familia es normal, el único oveja negra de la familia he sido yo, comencé fumando tabaco, al poco hachís y pronto cocaína, abandoné el colegio y comencé a robar, dejé de asistir al Proyecto Hombre, me hacía falta estar encerrado.

El que es drogadicto lo es siempre, que consuma o no, no quiere decir que dejes de ser drogadicto. Cuando llevas tiempo aquí, comienzas a adquirir seguridad para salir y verte con fuerzas para no consumir. El futuro es incierto, todos queremos trabajar, el pensamiento es uno y al final cuando estemos en la calle es cuando se verá el día a día en la calle” (Caso 2, 20 años). 
Caso 3: Joven de 20 años. Con medida de internamiento en Centro terapéutico por un delito de robo con fuerza. Había cumplido seis medidas en Centro cerrado y tiene medidas por cumplir. Abandono la escuela cuando cursaba el primer curso de la ESO. Pertenece a una familia estructurada y colaboradora y le visita en el Centro. El padre es pensionista y la madre es limpiadora es el tercero de 4 hermanos y la familia reside en una zona rural problemática. Las adicciones que presentan son hachís, coca, pastillas, alcohol heroína. La valoración del Centro es que tiene perspectivas de reinserción.

“Comencé a consumir cocaína a los 12 años con otros chicos mayores que yo, la primera vez que entre en un Centro tenía 16 años, fue "un palo" para mi familia porque es una familia normal sin ningún tipo de problemas. Mis hermanos están todos trabajando con buenos trabajos, casados, soy el único que está soltero. Me dejé llevar mucho por los demás, los amigos te influyen, no hacía nada, estaba todo el día con la moto, engañaba a mi madre y al resto de mi familia, cuando yo le decía me voy a quedar en casa de ...(..) fulanito, mi madre qué iba a pensar que su hijo iba a estar por ahí poniéndose hasta arriba de cocaína, estaba en la calle tirado, es lo último que a una madre se le pasa por la cabeza y hay muchos chicos en esta circunstancia. Hasta que estaba en una situación deplorable y con problemas con la justicia ellos no admitieron mi problema, estuve en Proyecto Hombre durante un mes que abandoné porque sabía que aquello no servía para nada, ya que yo seguía consumiendo. A la familia les tenía cansados, mis hermanos estaban muy pendientes de mi, pero le buscaba las vueltas y me peleaba con ellos y seguía haciendo lo que quería". "Comencé a consumir por curiosidad y querer saber lo que es eso y cuando lo pruebas te gusta, no creo que sea por educación, somos 4 hermanos y 2 tenemos problemas y nos hemos enganchado. Al probar corres el riesgo de tener problemas. La curiosidad es lo peor y si además te juntas con gente mayor, ya sabes..." (Caso 3, 20 años).

Caso 4: Joven de 18 años, cumpliendo medida de internamiento en Centro terapéutico por un delito de robo con fuerza, ha cumplido medidas anteriores. Abandonó la escolaridad obligatoria cuando cursaba la educación primaria y trabajando de ayudante de pescadería. Pertenece a una familia desestructurada mono-parental, recibe visitas en el Centro aunque la familia no es colaboradora. El padre es pensionista y la madre se dedica a sus labores; ocupa el segundo lugar de tres hermanos. Los padres son toxicómanos rehabilitados. La familia reside en una barriada urbana marginal. Presenta adicción a hachís, cocaína, sustancias sintéticas, alcohol y heroína. Las actividades las realiza en el Centro. Desde el Centro valoran de forma positiva una reinserción futura. Ha vivido su socialización primaria en el consumo de estupefacientes habiendo repetido este círculo vicioso tan pernicioso.

"Yo también he visto de todo en mi casa, mis padres vendían drogas, mis tíos también con 8 años comencé a fumar tabaco, con 10 consumía drogas y robaba para conseguirla. Se separan mis padres que eran consumidores de todo tipo de drogas y vendían empezando a consumir en exceso jestaba enganchado!, quisieron internarme en un colegio, pero no aguante me daba igual todo, me buscaba la vida robando, venga detenciones y mi padre me pegaba y encerraba pero me escapaba y me iba con mi colegas y no aparecía por mi casa robando, me ingresaron en un Centro y en los permisos consumía, hasta que he ingresado en este Centro, estoy mejor, comprendo que lo que llevaba no era vida. Toda familia ha cumplido condena por tráfico de drogas, mi padre 8 años, también mis tíos.... (..)..”(Caso 4, 18 años) 
Caso 5: Joven de 21 años, cumpliendo medida en un Centro terapéutico por un delito de robo con violencia. Abandono la escolaridad cuando cursaba el primer año de la ESO. Pertenece a una familia estructurada que le visita en el centro. El padre trabaja en la empresa de Astilleros, ocupa el segundo lugar de 3 hermanos. La familia reside en una barriada urbana problemática. Los hermanos también tienen problemas con la justicia. Las actividades las realiza en el centro. Presenta adicción a hachís, cocaína, sustancias sintéticas y alcohol. Respecto a la valoración del centro sobre la probabilidad de futura reinserción la califican como positiva.

"Empecé muy joven, en la familia existía consumo, hace 7 años la policía hizo hasta un registro en mi casa, antes mi familia traficaba, mis tíos fallecieron. Desde muy pequeño lo que he visto ha sido droga, con 12 años abandoné el colegio y comencé a pasar todo el tiempo con los amigos y a consumir, quería dar a respetar a los problemas de la casa cada vez consumía mas, "estaba siempre colocao", quería quitarme las paranoias de la familia, consumía hachís y casi del tirón cocaína. A los 14 años tuvo un accidente de moto con traumatismo cráneo encefálico, "me peleé con mi novia y me comí una caja de pastillas, me quedé dormido en la moto y me abrí la cabeza", cuando sali del hospital segui igual, he traficado, yendo a Ceuta a por hachís, ahora ve la calle de otra forma, antes no tenía conciencia de la situación que tenia, me daba igual todo, quería vivir la vida fácil. Mis padres nunca han podido conmigo, con 16 años no paraba nunca en la casa, tenía una casa alquila con una chavala y desde los 14 años se me busqué la vida, cuando no tenía dinero me dediqué a robar y traficar, con 14 años me detuvieron en Ceuta con 500 gramos de hachís y a los 16 años con $750 \mathrm{gr}$. Con mi familia problemas no he tenido, pero el consumo de drogas te hunde, cuando uno se quiere dar cuenta no puede salir" (Caso 5, 21 años).

Las poli-toxicomanías conllevan, más temprano que tarde, problemas con la justicia. Solo reconocen sus dificultades en el proceso de rehabilitación pero la espada de "Damocles" siempre está presente.

"Desde que estamos en el Centro y no consumimos, pensamos de otra forma, vemos las cosas de forma diferente. Queremos tener una vida normalizada, tenemos que mirar por nosotros y por la familia. No quiero verme en prisión. Sales a la calle y ves los que consumen y están.......... (..)” (Caso 4, 18 años).

\section{A MODO DE CONCLUSIÓN}

Son jóvenes todos ya mayores de edad, que con 15 años ya habían dejado todos de estudiar y, en general, faltaban a clase relatando que todos los días salían de su casa para ir al colegio y no llegaban, cuando llegaba la hora de salir de clase volvían a casa como si hubieran estado en el colegio, situación que duraba mientras que la familia lo descubría o el colegio se ponía en contacto con los padres y se activaba el protocolo de absentismo. Arrastraban retraso. Eran expulsados, se aburrían. Los padres llega un momento que se cansan de la situación. Reconocen que en el juicio el juez/a le dijo que si hubieran estado en el colegio no hubieran ingresado en un Centro. Saben que la inserción laboral es muy importante; es por lo que llegas a cambiar de verdad. 
En general, alaban el Centro y su funcionamiento y lo que más les gusta del Centro es la forma en que les conminan a cambiar para poder trabajar, ayudándoles para evitar que vayan a prisión.

La educación que ellos darían a sus hijos sería evitar que pasaran por su situación, les alejarían de las drogas. Les enseñarían lo que ellos no han aprendido en el colegio y les obligarían a ir, alejarles de los amigos que les pueden influir de forma negativa.

En general piensan que el centro les ha hecho cambiar de opinión, la idea es tener una meta en la calle para buscar un trabajo y no volver al mismo ambiente, tener más formación para poder trabajar.

Generalmente buscan los amigos del colegio en similares circunstancias que ellos (fracaso escolar, consumo estupefacientes, mucho tiempo libre), comienzan a robar. Aunque no achacan totalmente a los amigos sus circunstancias, aunque imitaban sus conductas, el consumo es un problema que está en la calle. Consideran que debería existir mayor control por parte de las autoridades y estar más penado en la ley. Son conscientes que el consumo es un problema que les destroza, pero cuando estás dentro no eres consciente ni tampoco que cuando salgan sean capaces de no volver a consumir nunca más.

El consumo y los amigo de riesgo les arrastran a sus formas de consumir y vivir para tener compañía en sus fechorías, se dan cuenta tarde, aunque es difícil entender a veces antes de que pase algo a los que te aconsejan bien, es decir, nadie escarmienta en cabeza ajena.

En general acataban pocas normas y eludían las que les imponían, convirtiendo la situación en un gran problema y con grandes conflictos de convivencia.

\section{BIBLIOGRAFÍA}

CAMPS, V. (2008), Creer en la educación. La asignatura pendiente, Madrid, Ediciones Península. CÓDIGO CIVIL. Real Decreto (1889), de 24 julio.

CÓDIGO PENAL (1995), de 23 de noviembre, Ley Orgánica, España, Civitas.

CONSTITUCIÓN ESPAÑOLA (1978), de 27 de diciembre.

CONVENCIÓN DE NACIONES UNIDAS (1989), Estupefacientes.

CONVENCIÓN DE NACIONES UNIDAS (2008), Estupefacientes.

ELZO, J. (2003), "Drogas y Escuela", VI Evolución del consumo de drogas en escolares donostiarra (1981-2003). San Sebastián, Escuela de Trabajo social.

ELZO, J. (2004), "Los valores de la sociedad ante las drogas: dos investigaciones recientes". En Kahler, H.; Abeijon Merchan, J.A. Drogas, sociedad y ley: avances en drogodependencias, Bilbao, U. Deusto, pp. 43-76.

GALLARDO FERNÁNDEZ, C; NIETO-MORALES, C. (2010), El fracaso escolar y la exclusión social de los jóvenes en el Polígono Sur de Sevilla. Causas, actuaciones, estrategias y perspectivas futuras, Sevilla, Asociación EntreAmigos.

GONZÁLEZ RESA, A. (2009), "Fundamentos y claves para el futuro de los Servicios Sociales y el Trabajo Social", Barataria. Revista Castellano-Manchega de Ciencias Sociales nº.10 pp. 191-207.

IBÁÑEZ, J. (1986), Más allá de la sociología. El grupo de discusión: técnica y crítica, Madrid, Siglo XXI.

INFORME DEL PLAN NACIONAL DE DROGAS (2007), Ministerio de Sanidad. Consulta 27 Junio de 2012 http://www.pnsd.msc.es/Categoria2/publica/pdf/InformeCannabis.pdf.

INFORME MUNDIAL SOBRE DROGAS (2008), Organización Naciones Unidas.

KRAMER, J.F.; CAMERON, D.C. (1975), Manual sobre dependencia de las drogas, Ginebra, O.M.S.

LEY 13/2009, de 3 de noviembre, de reforma de la legislación Procesal, Implantación de la nueva Oficina judicial, entro en vigor el 4 de mayo de 2010. 
LEY 14 de septiembre de 1882, por el que se aprueba la Ley de Enjuiciamiento Criminal, Real Decreto. LEY 19/1993, 28 de diciembre, Sobre determinadas medidas de Prevención del blanqueo de capitales. LEY 19/2003, Utilización del sistema financiero para el blanqueo de capitales.

LEY ORGÁNICA DE RESPONSABILIDAD PENAL DEL MENOR (2000), De 13 de enero y (2006) de 4 de diciembre.

LÓPEZ BLASCO, A.; CACHÓN, L.; COMAS, D.; ANDREU, J.; AGUINAGA, J. y NAVARRETE, L. (2005), Informe Juventud en España 2004: Condiciones de vida y situación de los jóvenes, Madrid, Instituto de la Juventud.

LUENGO, M.A.; OTERO-LÓPEZ, J.M.; ROMERO, E. y GÓMEZ-FRAGUELA, J.A. (1996), "Efectos de la necesidad de búsqueda de sensaciones sobre la involucración en el consumo de drogas de los adolescentes", Análisis y Modificación de Conducta, 22 nº 86, 683-709.

NIETO-MORALES, C. (2005), "El delincuente juvenil sevillano", Sevilla, La Toga. pp. 15-29.

NIETO-MORALES, C. y GONZÁLEZ LARA, A.M. (2012), "Prevención y educación frente a la violencia intrafamiliar", en Nieto-Morales, C. (Coord.) (2012), La violencia intrafamiliar, menores, jóvenes y género, Barcelona, Bosch. pp. 139-147.

ORGANIZACIÓN GENERAL NACIONES UNIDAS (1989), Convención sobre los Derechos del Niño, de 20 de noviembre.

ORGANIZACIÓN NACIONES UNIDAS (2010), Junta Internacional de Fiscalización de Estupefacientes, de 24 de febrero, en http://www.incb.org/pdf/s/ar/2005/incb_report_2005_full_es.pdf visitado $28 / 6 / 2012$.

ORGANIZACIÓN NACIONES UNIDAS (2008), Oficina de las Naciones Unidas contra la Droga y el Delito (ONUDD), en http://europa.eu/legislation_summaries/justice_freedom_security/combating_dru gs/c22569 es.htm, visitado 28/6/2012.

OTERO PÉREZ, A. (2009) "Tendencias en el consumo de drogas en la juventud española y efectos sobre su salud", Revista Estudios de Juventud n ${ }^{\circ} 84$. INJUVE. Ministerio de Igualdad, pp. 156, en http://www.feafes.com/NR/rdonlyres/43A2F0CD-867A-4E04-A5B0-A1F07545A32/28416/Lasaludme ntaldelaspersonasjvenesenEspaa.pdf, visitado 28/6/2012.

ROMEO CASANOVA, C. M. (2006), La conducción bajo la influencia de bebidas alcohólicas. Drogas tóxicas, estupefacientes y sustancias psicotrópicas, Granada, Comares.

RUIZ VEGUILLA, M (2008), Factores genéticos y ambientales asociados a la psicosis no afectiva de inicio en la infancia y adolescencia, Granada, Universidad.

TABERNER GÜAS, J (2012), Sociología y educación. El sistema educativo en las sociedades modernas. Funciones, cambios y conflictos, Madrid, Tecnos.

TOURAIN, A. (2006), "Los movimientos sociales”, Colombia, Revista de Sociología. pp. 255-278.

\section{Breve currículo:}

\section{Concepción Nieto Morales}

Doctora en Sociología. Ejerce de trabajadora social en los Equipos Técnicos de la Fiscalía de menores. Imparte clase como profesora asociada en el Departamento de Trabajo Social de la Universidad Pablo Olavide de Sevilla. Las principales líneas de investigación son menores, justicia, intervención y personas mayores. 